\title{
Lambert-Eaton myasthenic syndrome without anti-calcium channel antibody: adverse effect of calcium antagonist diltiazem
}

\author{
S Ueno, Y Hara
}

\begin{abstract}
A 59 year old man with ischaemic heart disease, developed the clinical and electromyographic changes of the LambertEaton myasthenic syndrome after taking calcium antagonist, diltiazem. The symptoms appeared periodically with a rise and fall in serum level of diltiazem. Extensive search was made for systemic neoplasms and autoimmune diseases without success. Serum antibody to voltage operated calcium channel was not detected.
\end{abstract}

Lambert-Eaton myasthenic syndrome (LEMS) is an autoimmune disorder associated with malignant neoplasms and occasionally with immunological disorders. ${ }^{1-5}$ Autoantibodies interfere with acetylcholine (ACh) release from the motor nerve terminals by affecting the function of the voltage operated calcium channel (VOCC). ${ }^{67}$ Although organic VOCC antagonists including verapamil and diltiazem, have been shown to reduce $\mathrm{ACh}$ release in in vitro experiments, ${ }^{89}$ only one paper has been published on the adverse effect of the antagonist verapamil on neuromuscular transmission in a human subject. ${ }^{10}$ The VOCC blocking agent, diltiazem, is commonly used in the treatment of supraventricular arrhythmias and ischaemic heart disease. We describe a case which showed LEMS after taking diltiazem.

\section{Case report}

Since the age of 55, the patient had been given diltiazem (90 mg/day) for ischaemic heart disease. At the age of 56 , his voice became nasal. Muscle strength of the hands and proximal portions of the four extremities was decreased. The weakness peaked after rest or immediately after waking up in the morning, and the strength tended to improve transiently with brief exercise. Other complaints included dry mouth, decreased sweating, constipation and weight loss. One year after starting the drug, the man noted muscular weakness in the thighs. He had smoked 20 cigarettes per day for 37 years. On admission at the age of 59 , there was no abnormality in the movement of the eyes, eyelids, or facial muscles. Sensory examination was normal. Withdrawal of diltiazem was followed by improvement of the nasal voice and muscular weakness. Both symptoms reappeared with diltiazem but again decreased after withdrawal. The serum concentration of diltiazem correlated with the severity of the muscular weakness. Signs of muscular weakness did not change with edrophonium chloride, but were relieved after five days of guanidine (300 mg/day).

ELECTROMYOGRAPHIC STUDY

Electrodiagnostic studies on the abductor pollicis brevis demonstrated a small compound muscle action potential in response to a single supramaximal stimulation of the median nerve $(2.5 \mathrm{mV}$, normal $>5.0 \mathrm{mV})$. At $5 \mathrm{~Hz}$ repetitive nerve stimulation, $11 \%$ decrement, and postexercise facilitation and exhaustion occurred in the muscle (fig A, B, C). There was a $270 \%$ facilitation at $30 \mathrm{~Hz}$ supramaximal stimulation. (fig D). Improvement in the incremental response at $30 \mathrm{~Hz}$ stimulation $(57 \%$ facilitation) was observed after withdrawal of diltiazem (fig E).

\section{LABORATORY EXAMINATION}

Full blood counts and routine laboratory tests for hepatic and renal functions gave. normal values. At the time of maximal weakness, serum electrolytes including potassium, magnesium and calcium were of normal levels. Immunological tests gave a negative reaction for anti-nuclear factor, LE cells and RA. Antiskeletal muscle antibody, anti-ACh receptor antibody, oligoclonal IgG and myelin basic protein were not detected. Tumour markers including $a \mathrm{FP}, \mathrm{CEA}$, elastase I, CA 125 were all negative. Serum levels of thyroid hormones, anti-thyroglobulin test, and microsome test gave normal values. Chest $x$ ray, whole body

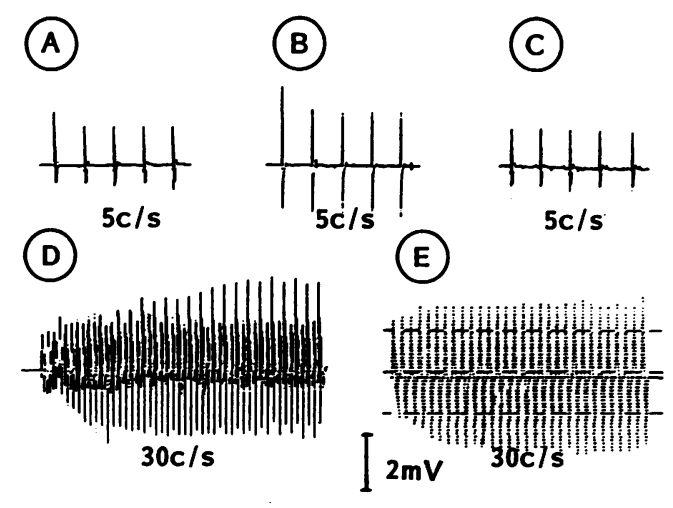

Figure Compound muscle action potentials to repetitive stimulations of the median nerve. 
CT scanning, brain MRI and $67 \mathrm{Ga}$ scanning of the body and $99 \mathrm{~m}$ Tc scanning of bone gave normal findings.

SERUM ANTIBODY TO VOCC

The antibody was measured by the method described by Sher et al. ${ }^{11}$ VOCCs of IMR 32 cells (American Type Culture Collection, Rockville, USA) labelled with $\omega$-Conotoxin (Amersham) were used as the source of the antigen in the immunoprecipitation assay. Increasing amounts (from 1 to $10 \mu \mathrm{l}$ in triplicate) of each sample of human serum were added and left in the presence of the antigen overnight at $4^{\circ} \mathrm{C}$. A sufficient amount of antihuman IgG was added to each tube and the samples were centrifuged. The resulting pellets were washed twice and the radioactivities of the pellets were counted in a gamma counter. Serum from the first Japanese case in which anti-calcium channel antibodies have been previously detected by radioimmunoassay $(47 \mathrm{pM})^{12}$ was used as a positive control in our assay system. Negative control sera were obtained from 10 healthy subjects. AntiVOCC antibody in our case $(1.2 \mathrm{pM})$ was not of a significant level (normal $>5 \cdot 2 \mathrm{pM}$ ).

\section{Discussion}

This patient fulfilled the clinical, pharmacological and electrodiagnostic criteria for LEMS; proximal muscular weakness, amplitude of low compound muscle action potential, decremental response at low stimulus rates, abnormal facilitation after exercise and beneficial effect of guanidine. The abnormality in classical LEMS usually spares bulbar musculatures. The most significant problem in this case, however, was the nasal voice, because the patient worked as a telephone operator. On withdrawal of diltiazem, the serum level decreased, and subsequently the muscular weakness and electromyographic findings improved. This correlation was reproducible in a second trial with diltiazem. All these facts showed the adverse effect of diltiazem on neuromuscular transmission in this case.

VOCCs are divided into three categories, $T$, $\mathrm{N}$, and $\mathrm{L}$ defined in terms of their activation potentials and rate of inactivation. ${ }^{13}$ It is not yet fully clarified which types of channels are involved in transmitter release and are blocked in patients with LEMS. Several authors have reported that the $\mathrm{L}$ type channel is not involved in the transmission in mammals, ${ }^{14}{ }^{15}$ but others have stated that $\mathbf{N}$ and $\mathrm{L}$ coexist in the presynaptic and both may be involved in the neuromuscular transmission. ${ }^{89}$ Peers et al showed that IgG from LEMS patients acted on $L$ type calcium channel of hybrid cells and suggested the immunological similarities between the channel and presynaptic membrane in LEMS patients. ${ }^{7}$
The data obtained from our case strongly suggests that diltiazem, antagonist of $L$ type calcium channel, impaired neuromuscular transmission. The possible explanation for the blocking mechanism was that diltiazem reduced channel lifetime and the frequency of channel activation, leading to a decrease in the safety margin for neuromuscular transmission. Such a decrease will become especially important when transmission is depressed by preexisting neuromuscular blocking agents. This explanation may agree with the data obtained from animal experiments. Low concentrations of verapamil and diltiazem increase potency of the neuromuscular blocking agents. ${ }^{1415}$

Although we have not found the neoplasms or autoimmune disorders in which anti-VOCC antibodies are occasionally produced, a possibility that the undefined factors interfere with the neuromuscular transmission in this patient is not excluded. If such factors pre-existed, the diltiazem could enhance the neuromuscular transmission block by reducing $\mathrm{ACh}$ release from the motor nerve terminals. The relationship to the clinical worsening in this case and the theoretical danger of calcium channel blockade appear to support this hypothesis.

This study was supported by a grant from the Neuroimmunological Disease Research Committee of Japan.

1 Lambert EH, Eaton LM, Rooke ED. Defect of neuromuscular conduction associated with malignant neoplasms. Am $\mathcal{f}$ Physiol 1956;187:612-3.

2 Lennon VA Lambert EH, Whittingham S, Fairbanks U. Autoimmunity in the Lambert-Eaton myasthenic synAutoimmunity in the Lambert-Eaton

3 O'Neil JH, Murray NMF, Newsom-Davis J. The LambertEaton myasthenic syndrome. A review of 50 cases. Brain 1988;111:577-96.

4 Hughes RI, Katirtji MB. The Eaton-Lambert (myasthenic) syndrome in association with systemic lupus erythematosus. Arch Neurol 1986;43:1186-7.

5 Lang B, Newsom-Davis J, Wray D, Vincent A, Murray N Autoimmune aetiology for myasthenic (Lambert-Eaton) syndrome. Lancet 1981;2:224-6.

6 Fukunaga N, Engel AG, Lang B, Newsom-Davis J, Vincent A. Passive transfer of Lambert-Eaton myasthenic syndrome IgG from man to mouse depletes the presynaptic membrane active zones. Proc Natl Acad Sci USA 1983;80:7636-40.

7 Peers C, Lang B, Newsom-Davis J, Wray DW. Selective action of myasthenic syndrome antibodies on calcium channels in a rodent neuroblastoma $\times$ glioma cell line. $\mathscr{f}$ Phys 1990;421:293-308.

8 Chang CC, Lin SO, Hong SJ, Chiou LC. Neuromuscular block by verapamil and diltiazem and inhibition of block by verapamil and diltiazem and inhib

9 Wachtel RE. Effects of diltiazem and verapamil on responses to acetylcholine. Br $\mathcal{F}$ Pharmac 1987;92:561-6.

$10 \mathrm{Krendel}$ DA, Hopkins LC. Adverse effect of verapamil in a patient with the Lambert-Eaton syndrome. Muscle Nerve 1986;9:519-22.

11 Sher E, Gotti C, Canal N, Scoppettac C, Piccolo G, Evolt A, Clementi F. Specificity of calcium channel autoantibodies in Lambert-Eaton myasthenic syndrome. Lancet 1989;16:640-3

12 Yamada A, Takeuchi H, Touge T, Miki H, Kuwabara $H$. Eaton-Lambert syndrome manifested by respiratory failure associated with small cell carcinoma of the lung. Clin Neurol 1990;30:780-3.

13 Tsien RW, Lipscombe D, Madison DV, Bley KR, Fox AP Multiple types of neuronal calcium channels and their Multiple types of neuronal calcium channels and the

14 Bikhazi GB, Leung I, Foldes FF. Interaction of neuromuscular blocking agents with calcium channel blockromuscular blocking agents with

15 Bikhazi GB, Leung I, Foldes FF. Ca-channel blockers increase potency of neuromuscular blocking agents in vivo. Anesthesiology 1983;59:A269. 